\title{
Association of Work Risk Factors and the Development of Cardiovascular Diseases: Protocol of a Systematic Review
}

Christian Moretti Anfossi ( $\sim$ chritian.anfossi.19@ucl.ac.uk)

University College London https://orcid.org/0000-0001-6366-6561

Christian Tobar Fredes

Mutual de Seguridad Asesorías S.A.

Eduardo Quiñelen Rojas

Universidad Metropolitana de Ciencias de la Educación

Jamie Ross

University College London

Jenny Head

University College London

Annie Britton

University College London

\section{Study protocol}

Keywords: Systematic Review, Cardiovascular Disease, Job Strain, Effort-Reward Imbalance, Overtime Work, Job Insecurity, Shift Work, Occupational Noise.

Posted Date: June 17th, 2021

DOl: https://doi.org/10.21203/rs.3.rs-608667/v1

License: (9) This work is licensed under a Creative Commons Attribution 4.0 International License. Read Full License 


\section{Abstract}

Background: Cardiovascular Diseases (CVDs) are the number one cause of death, representing $31.8 \%$ of all global deaths. Several lifestyle behaviours are associated with the development and clinical manifestation of CVDs, however, these behavioural risk factors by themselves do not fully explain the population burden of CVDs. There is increasing recognition that working conditions and risk factors of the work environment are associated with health, including the development of CVDs. We will systematically review observational studies of adults exposed to work risk factors and their association with CVDs.

Methods: We will follow the Navigation Guide framework. We will include cohort and case-control studies. The population will be adults of working age (18-65). The exposure will include six categories of work exposure: job strain, effort-reward imbalance, long working hours, job insecurity, shift work and occupational noise; the comparator will be the unexposed group or specified control group. The outcomes will be cerebrovascular diseases, ischaemic heart disease and hypertensive diseases. Published and unpublished studies will be included. The selection, data extraction, risk of bias assessment, quality assessment and strength of evidence will be carried out by two reviewers independently and disagreements will be solved by a third. Due to the diversity in the populations and exposures in the studies, the synthesis of the results, the quality and the strength of the evidence will be done by a synthesis without meta-analysis (SWiM), following the SWiM reporting guideline.

Discussion: This systematic review will identify and synthesise the evidence for the association between work risk factors and risk of CVDs. This work will underpin and inform a broader objective to examine the effectiveness of interventions to minimise the effects of risk factors for CVDs in workplaces, with the final aim of informing occupational health policies in the future.

\section{Systematic review registration: PROSPERO CRD42020179972.}

\section{Background}

Cardiovascular Diseases (CVDs), defined by the World Health Organization (WHO) as a "group of disorders of the heart and blood vessels", are the number one cause of death (1), representing $31.8 \%$ of all global deaths (2). This high disease prevalence has a significant economic impact for nations, but also for employers since CVDs are the most costly to companies in terms of lost productivity as a result of disability and death (3). It is estimated that CVDs in the European Union cost 169 billion euros per year, with lost productivity representing 21 per cent of these costs (3).

Several lifestyle behaviours are associated with the development and clinical manifestation of CVDs (4). The main ones are an unhealthy diet, physical inactivity, tobacco use and harmful use of alcohol (1). However, behavioural risk factors by themselves do not fully explain the population burden of CVDs (4); there are other determinants, like socioeconomic and psychosocial factors and genetics (1). 
Furthermore, we have experienced a rapid global economic growth that has resulted in a variety of potentially significant occupational exposures that pose a risk for health (5). During the 19th century, the "hygienist" approach stated that the effects of work on health were due only to the lifestyle and habits of workers and the problems of urban insalubrity, taking away all the responsibility of companies (6). Nowadays, the development of international policies in human, social and labour rights has exposed the importance of working conditions and risk factors of the work environment, and how they represent a threat to health, safety and well-being (7). Previous research has shown associations between working conditions and risk of CVDs (5), so much so that in Japanese there exists a word to describe sudden death because of overwork, "Karoshi" (4), which is mainly the result of acute cardiovascular events (8)

One of the key underlying determinants of CVDs is stress (1) and one of the most studied sources of acute and chronic stress is work (5). Traditionally it was believed that stress and heart health were connected by associated behaviors - such as smoking, drinking alcohol or eating fatty foods, however, there are now well described physiological pathways (9)(10). The pathways (9) are complex and involve activation of the hypothalamic-pituitary-adrenocortical axis (HPA) and the sympathetic-adrenal-medullary system (SAM).

To understand the relationship between work stress and CDVs, researchers have expanded the concept of work stress beyond the characteristics of the work task to encompass organizational factors, generating conceptual models/approaches that indicate risk conditions where those factors interact (11) (5). There are six common working conditions associated with psychosocial risk factors related to CVDs described in the literature: job strain, effort-reward imbalance, long working hours, job insecurity, shift work and occupational noise (11)

\section{Job strain}

According to Karasek's model, job strain is the consequence of a combination of high job demands and low individual control over those demands (4) (11). The "job demand/control" hypothesis has been tested in different populations and has been shown to predict CVDs or mortality (3)(5).

\section{Effort-reward imbalance}

In Siegrist's model, the mismatch between high effort, low reward, and the individuals' exhaustive coping style (or overcommitment) leads to adverse health effects, as it violates core expectations about reciprocity and adequate exchange at work (11). It has been shown that this model has a significant ability to predict CVDs (4).

\section{Long working hours}

It is recognized that long working hours represent a danger to the health of workers and their families (12), including strong evidence to suggest that long working hours increases CVDs risk (11) (5).

\section{Job insecurity:}


There is evidence that high levels of job insecurity are associated with risk of incident coronary heart diseases. It is believed that this association is partly explained by poorer socioeconomic circumstances and less favorable risk factor profiles among people with job insecurity (11). An analysis of 22,400 employees showed that cardiovascular mortality was 2.0 (95\% confidence interval 1.0 to 3.9 ) times higher after major downsizing than after no downsizing (13).

\section{Shift work:}

Shift work, defined as "work occurring outside typical daytime working hours", is associated with an increased risk of diseases (14), such as CVDs (15). For example, night shift work produces a misalignment of the endogenous circadian timing system, which in turn is associated with alterations in a wide range of physiological parameters risky for CVDs (16) (15).

\section{Occupational noise:}

There is much evidence to suggest that occupational noise impacts on morbidity and mortality from CVDs, primarily through causing a high-stress level and response (17) (18). Exposure to certain levels of noise can lead to biochemical, physiological and psychosocial alterations, interfering with the gastroenteric system, endocrine system, central nervous system and psychological alterations (19), all of them related directly or indirectly with the pathogenesis of CVDs.

Although there are many studies on the association of working factors and CVDs, the causal connection is still subject to debate and poorly understood (11). Furthermore, most of the studies focus on one kind of risk factor (such as psychosocial) and one disease as the outcome (such as stroke or myocardial infarction).

\section{Methods}

\section{Aim of the study}

The aim of this study is to synthesise the evidence about the association of work risk factors and CVDs by performing a systematic review of observational studies of adults exposed to job strain, effort-reward imbalance, long working hours, job insecurity, shift work and occupational noise to assess the association of these work risk factors and the development of cerebrovascular diseases, ischaemic heart diseases or hypertensive diseases.

This systematic review will underpin and inform a broader objective to examine the effectiveness of interventions to minimise the effects of risk factors for CVDs in workplaces, with the final aim of informing occupational health policies in the future.

\section{Study design}

Due to the complexity and ethical considerations, human observational studies are the gold standard of the evidence base in the field of environmental health (20), which includes studies of work risk factors. 
This is a problem if systematic review methodologies as Cochrane and the Grading of Recommendations Assessment, Development, and Evaluation (GRADE) system are to be applied because they have been developed based on evaluation of randomized controlled clinical trials, considering human observational studies to be "low" quality evidence (20). That is the reason why our systematic review will be carried out following the Navigation Guide framework, which is a systematic and rigorous approach to research synthesis, based on best practices in the evaluation of information in evidence-based medicine and environmental health (20) (see Figure 1). It assigns a "moderate" quality rating to human observational studies and allows combination of diverse evidence streams (20).

This protocol adheres to the recommendation of the Preferred Reporting Items for Systematic Reviews and Meta-analysis Protocols guidelines, published in 2015 (PRISMA-P 2015) (21) (see Additional file 1: PRISMA-P 2015 Checklist) and it has been registered on the International Prospective Register of Systematic Reviews (PROSPERO) (CRD42020179972). All the updates will be registered on PROSPERO and reported in the systematic review itself.

\section{Eligibility criteria for study selection}

Following the first step of the Navigation Guide, we will specify the study question using the Population, Exposure, Comparators and Outcomes (PECO) framework (20). A summary of the PECO statement of this study in Table 1.

\section{Table 1: PECO statement}

\begin{tabular}{|ll|}
\hline Population & Adults in working age \\
\hline Exposure & Work risk factors \\
\hline Comparator & Unexposed group or control group specified \\
\hline Outcomes & Cardiovascular diseases \\
\hline
\end{tabular}

Details on the components of the PECO statement will be addressed in the following sections.

\section{Types of studies}

We will include quantitative observational research studies, specifically cohort studies (both prospective and retrospective) and case-control studies.

We will exclude randomized controlled trials, quasi-experimental trials, cross-over controlled trials, controlled trials without randomization, single-case studies, review articles, short communications, letters with insufficient information to analyse the results, guidelines, dissertations, qualitative studies, scientific conference abstracts and studies on animals.

\section{Population}


Inclusion: Studies of adults of working age at the baseline (18-65 years), working in the formal economy.

Exclusion: Studies of children (aged $<18$ years), unpaid domestic workers will be excluded. Individuals with previous CVDs.

No restrictions will be imposed on the setting of recruitment.

\section{Exposures}

The working conditions and risk factors that will be included are:

- Job Strain.

- Effort-Reward Imbalance.

- Long Working Hours.

- Job Insecurity.

- Shift Work.

- Occupational Noise.

For eligibility criteria, we will include studies which apply descriptions of the work conditions and risk factors according to our definitions (see Additional file 2: Definition of each Working Condition and Risk Factor for Inclusion).

\section{Comparator}

Unexposed group or control group specified, depending on the risk factor assessed.

\section{Outcomes}

Development of a cardiovascular disease according to our definitions in Table 2, which follows the International Classification of Diseases 11th Revision, 2019 (ICD11) and its equivalence in the 10th Revision (ICD10), within the following groups of diseases:

- Cerebrovascular diseases.

- Ischaemic heart disease.

- Hypertensive diseases.

Table 2: Inclusion criteria for diseases (International Classification of Diseases 11/10th Revisions, 2019) (22). 


\begin{tabular}{|c|c|c|c|}
\hline $\begin{array}{l}\text { Group of diseases } \\
\text { (Outcomes) }\end{array}$ & Diseases included & ICD11 Codes & ICD10 Codes \\
\hline \multirow[t]{7}{*}{$\begin{array}{l}\text { Cerebrovascular } \\
\text { diseases }\end{array}$} & Intracranial haemorrhage & $\begin{array}{l}\text { 8B00-8B03, } \\
8 \mathrm{~B} 0 \mathrm{Z}\end{array}$ & $160-169$ \\
\hline & Cerebral ischaemia & $\begin{array}{l}\text { 8B10, 8B11, } \\
\text { 8B1Y, 8B1Z }\end{array}$ & $\begin{array}{l}\text { G45, } 163 \\
167.8,167.8\end{array}$ \\
\hline & $\begin{array}{l}\text { Stroke not known if ischaemic or } \\
\text { haemorrhagic }\end{array}$ & $8 B 20$ & 164 \\
\hline & $\begin{array}{l}\text { Cerebrovascular disease with no acute } \\
\text { cerebral symptom }\end{array}$ & 8B21 & $160-169$ \\
\hline & $\begin{array}{l}\text { Certain specified cerebrovascular } \\
\text { diseases }\end{array}$ & $8 B 22$ & 167 \\
\hline & Hypoxic-ischaemic encephalopathy & 8B24 & G93.1 \\
\hline & Cerebrovascular diseases, unspecified & 8B2Z & $160-169$ \\
\hline \multirow[t]{3}{*}{$\begin{array}{l}\text { Ischaemic heart } \\
\text { disease }\end{array}$} & Acute ischaemic heart disease & $\begin{array}{l}\text { BA40-BA43, } \\
\text { BA4Z }\end{array}$ & $|20-| 25$ \\
\hline & Chronic ischaemic heart disease & $\begin{array}{l}\text { BA50, BA51, } \\
\text { BA5Z }\end{array}$ & $\begin{array}{l}125.2,125.5 \\
125\end{array}$ \\
\hline & Ischaemic heart diseases, unspecified & BA6Z & $|20-| 25$ \\
\hline \multirow{2}{*}{$\begin{array}{l}\text { Hypertensive } \\
\text { diseases }\end{array}$} & Essential hypertension & BA00 & 110 \\
\hline & Hypertensive heart disease & BA01 & 111 \\
\hline
\end{tabular}

For studies that applied older revisions of the International Classification of Diseases, the equivalences of the diseases will be used according to the WHO references.

We have excluded cardiovascular diseases with strong congenital evidence and diseases secondary to others, such as cerebrovascular abnormalities, late effects of cerebrovascular disease, vascular syndromes of brain in cerebrovascular diseases, certain current complications following acute myocardial infarction, hypertensive renal disease and secondary hypertension. Diseases of intracranial, extracranial and coronary arteries have been excluded because we are focusing on their effects which are included in our list of cerebrovascular diseases and ischaemic heart diseases.

For eligibility of measurement of cardiovascular disease, we will consider the following sources used by the World Health Organisation (WHO) and the International Labour Office (ILO) in their latest protocols to systematically review work-related burden of disease and injury (17) (23):

- Diagnosis by a medical doctor with images.

- Hospital discharge registry. 
- Other relevant administrative data (for example, absence record due to illness or disability with a diagnosis certified by a medical doctor).

- Treatment registration data for an eligible cardiovascular disease (including both drugs and medical procedures from register data).

- Medically certified cause of death.

Self- report measures of cardiovascular disease will not be considered to avoid bias in the measurement.

Specifically for hypertension, we will include only studies where the diagnostic was done after two or more measures on different days to avoid confusions with ambulatory hypertension.

\section{Search strategy}

Following the Navigation Guide recommendations (20) and to avoid publication bias, the first author will conduct comprehensive literature searches using electronic academic databases for potentially relevant records from published and unpublished studies.

For published studies, the following electronic academic databases will be used:

- Ovid Embase (1974 to 26 May 2020).

- Ovid Medline (1946 to 26 May 2020).

- PubMed (1946 to 26 May 2020).

- Scopus (1788 to 26 May 2020).

- Web of Science (1900 to 26 May 2020).

- Ovid APA Psyclnfo (1806 to 26 May 2020).

(See Additional file 3: Draft of Search Strategy in Ovid Embase as an example)

For unpublished studies we will include grey literature from these electronic grey literature databases:

- OpenGrey (http://www.opengrey.eu/).

- Open Thesis (http://www.openthesis.org).

- Grey Literature Report (http://greylit.org/).

- ProQuest Dissertations \& Theses Global ${ }^{\mathrm{TM}}$ (www.proquest.co.uk/products_pq/descriptions/pqdt.shtml).

Furthermore, we will include studies by hand searches from the following sources:

- Reference lists of included papers.

- Citing reference searching of included papers.

- Collections of the review authors. 
The search will be conducted in English words but without a language filter. If an article is written in a language other than English or Spanish, the document will be translated into one of these languages.

The downloaded references will be stored in the reference managers Endnote and Mendeley.

\section{Study selection}

To support all the stages of the systematic review, we will use the web-based software platform Covidence (24), where the study records will be uploaded. Duplicates will be identified and deleted. One reviewer will screen the titles and abstracts of the studies retrieved during the searches for relevance.

Two reviewers will then assess independently the full texts of articles identified as being potentially eligible for inclusion against the predefined criteria. Any discrepancies will be resolved by consensus or by a third reviewer.

We will illustrate the process of study selection at different stages in a PRISMA-based flow diagram (25).

If the full text of the article is not available in the databases used, we will conduct a more extensive search on the Internet. If after that search full text is not found, authors will be contacted and asked for the document. References, where full text and contact information are not available after the extensive search, will be excluded.

\section{Data extraction}

Data will be extracted by two reviewers independently and any discrepancies will be resolved by consensus or by a third reviewer. The extraction will be done using a data extraction form (See Additional file 4: Data Extraction Form), which will be designed and piloted by all the reviewers on 10 references before the data extraction.

For the data extraction of exposure and its measures, we will include specific fields for job strain, effortreward imbalance, long working hours, job insecurity, shift work and occupational noise, which will be applied according to the exposure assessed in the study in extraction.

Any change in the form will be updated in PROSPERO and registered in the systematic review itself.

Missing data will be requested from the authors by the email or phone provided in the principal study record.

\section{Quality of individual studies}

The risk of bias, defined as "characteristics of a study that can introduce systematic errors in the magnitude or direction of the results"(20), will be assessed for each selected article and will be done by two reviewers working in parallel by applying an adaptation of the Navigation Guide method for human studies tool (26)(23)(27), which judges the risk of bias by nine domains: 
1. Recruitment strategy

2. Blinding

3. Exposure assessment

4. Outcome assessment

5. Confounding (at least adjusted for age, sex and socioeconomic status)

6. Incomplete outcome data

7. Selective outcome reporting

8. Conflict of interest

9. Other sources of bias

For the risk of bias assessment we will use the instructions included in the Case Study 7 of Application of the Navigation Guide (26) (ongoing) for judging the risk of bias for human studies (Additional file 5:

\section{Instructions for Making Risk of Bias Determinations).}

Every domain will be graded as "Low Risk", "Probably Low Risk”, "Probably High Risk”, "High Risk” or "Not Applicable". The worst rating in any bias domain for any outcome will define the overall risk of bias at study level (17). Disagreements will be solved by consensus or by a third review author.

\section{Strategy for data synthesis}

This review will provide a description of the occupational risk factors related to cardiovascular diseases and their level of association in the development of cardiovascular diseases in working-age adults. Due to the diversity in the populations and exposures in the studies, the synthesis of the data will be done by a synthesis without meta-analysis (SWiM) (28).

The studies will be grouped by exposure (job strain, effort-reward imbalance, long working hours, job insecurity, shift work and occupational noise) and sub-divided by outcome (cerebrovascular diseases, ischaemic heart disease, hypertensive diseases).

To synthesise direction of the effects for each outcome we will apply the "vote counting based on direction" method (29) to have a big picture of the evidence to make decisions. This will be possible because we will include studies where the direction of effect is reported and because we expect the inclusion of a large number of reports (29). Following the recommendations of this method, we will create a standardized binary metric, where each effect estimate is categorized as showing the harm or benefit based on the observed direction of effect alone to finally compare the number of studies (votes) showing harm with the number showing benefit using a sign test (29). For the sign test, if there is no effect, the study will be part of the null hypothesis of no difference, following the Cochrane recommendations (30). Studies with "high risk of bias" and "probably high risk of bias" won't be included in the primary synthesis, however all the studies will be considered to draw the final conclusions about the strength of the evidence. 


\section{Quality of the overall body of evidence}

For grading the quality of evidence of each outcome by working condition and risk factor we will use the Navigation Guide tool (26) (Additional file 6: Instructions for Grading the Quality and Strength of

Evidence), which downgrades or upgrades the quality considering eight categories::

- Category 1. Quality of Study Limitations (Risk of Bias).

- Category 2. Indirectness of Evidence.

- Category 3. Inconsistency of Evidence.

- Category 4. Imprecision of Evidence.

- Category 5. Publication Bias.

- Category 6. Large Magnitude of Effect.

- Category 7. Dose-response.

- Category 8. Confounding Minimizes Effect.

The quality of evidence will be assessed by two reviewers working in parallel using the Navigation Guide quality of evidence assessment tool, grading the evidence in "high", "moderate" and "low" (26). Disagreements will be resolved by consensus or by a third review author.

\section{Strength of the evidence}

Finally, the overall strength of each body of evidence of each outcome by working condition and risk factor will be rated based on four considerations following the Navigation Guide criteria (26):

1. Quality of body of evidence.

2. Direction of effect.

3. Confidence in effect.

4. Other compelling attributes of the data that may influence certainty.

The assessment will be done by two reviewers applying the definitions in Additional File 6: Instructions for Grading the Quality and Strength of Evidence. The strength of the evidence will be rated in one of the following four categories:

- Sufficient evidence of toxicity/harmfulness

- Limited Evidence of Toxicity/harmfulness

- Inadequate Evidence of Toxicity/harmfulness

- Evidence of Lack of Toxicity/harmfulness 
Disagreements will be resolved by consensus or by a third review author.

\section{Discussion}

It is estimated that interventions in the workplace could reduce health care costs by $26 \%$ and reduce workers' compensation and disability management claims by $30 \%$ (31). However, optimal program delivery models have yet to be elucidated, therefore there is a need for additional research in this area (31). Specifically, for CVDs, while behavioural risk factors (unhealthy diet, physical inactivity, tobacco use and harmful use of alcohol) can be targeted with workplace interventions, there is evidence that there are stressful working conditions that can also be modified to reduce the risk.

This systematic review is part of broader research to develop guidelines about the most effective interventions to control the risk factors for CVDs in workplaces by including both traditional and work risk factor, with the final aim of informing occupational health policies in the future.

To achieve a better synchronization between public policies and scientific research, solid methods to evaluate the available scientific evidence are essential (26) and that is why we will systematically review the evidence of the association between work exposures and CVDs applying the Navigation Guide, which is a novel alternative, in terms of methodology and tools, for systematic reviews in occupational and environmental health.

Currently, systematic reviews are an essential source of evidence for decision makers (28) and providing this detailed protocol will improve the understanding, the transparency and the value of the review methodology, as well as detecting deviances from the original plan and minimising selective publication of the results (32).

\section{Strengths and Limitations of this Study}

Publication status and language bias will be reduced in this systematic review by including published and unpublished studies in all languages. Bias in the section, data extraction and analysis will be reduced by incorporating two researchers working in parallel independently at each of these stages. This predefined protocol has been also done to reduce the impact of reviewers' biases.

Limitations of the synthesis methods used and/or the groupings used in the synthesis and how these affect the conclusions will be reported in the systematic review itself.

\section{Abbreviations}

CVDs: Cardiovascular Diseases.; PRISMA: Preferred Reporting Items for Systematic Reviews and MetaAnalyses; PROSPERO: International Prospective Register of Systematic Reviews; GRADE: Grading of Recommendations Assessment, Development and Evaluation; PECO: Population, Exposure, Comparators, and Outcomes; ICD11: International Classification of Diseases, 11th Revision.; ICD10: International 
Classification of Diseases, 10th Revision; WHO: World Health Organisation; ILO: International Labour Office; HPA: Hypothalamic-Pituitary-Adrenocortical axis; SAM: Sympathetic-Adrenal-Medullary system; ISCO: The International Standard Classification of Occupations; JCQ: Job Content Questionnaire; ERI: Effort-reward imbalance.

\section{Declarations}

\section{Ethics approval and consent to participate}

Not applicable.

\section{Consent for publication}

Not applicable.

\section{Availability of data and materials}

Not applicable.

\section{Competing interests}

The authors declare that they have no competing interests.

\section{Funding}

This study it been funded by the Chilean National Agency for Research and Development (CONICYT), and the University College London (UCL). The CONICYT does not participate in the development of the review question, the data analysis or the summary and the presentation of results.

\section{Authors' contributions}

$\mathrm{CM}$ is the guarantor of the review.

$\mathrm{CM}$ presented the idea for the systematic review. $\mathrm{AB}$ contributed to the development of the idea.

$\mathrm{CM}$ worked with $\mathrm{AB}, \mathrm{JH}, \mathrm{JR}, \mathrm{CT}$ and $\mathrm{EQ}$ to define search strategy, study eligibility criteria, create a plan for study selection, data extraction, risk of bias assessment and graduate the quality and strength of evidence.

This manuscript was critically appraised and approved by all participating authors.

\section{Acknowledgements}

Not applicable. 


\section{References}

1. World Health Organization. Cardiovascular diseases (CVDs). https://www.who.int/news-room/factsheets/detail/cardiovascular-diseases-(cvds)]. (2017). Accessed 18 Dec 2019.

2. GBD Compare | IHME Viz Hub. https://vizhub.healthdata.org/gbd-compare/. Accessed 25 May 2020 May 25.

3. International Labour Office. SOLVE: integrating health promotion into workplace OSH policies: trainer's guide. International Labour Office Geneva. 2012. 1-346 p.

4. Neylon A, Canniffe C, Anand S, Kreatsoulas C, Blake GJ, Sugrue D, et al. A Global perspective on psychosocial risk factors for cardiovascular disease. Prog Cardiovasc Dis. 2013;55(6):574-81.

5. Gatchel R, Schultz I, editors. Handbook of Occupational Health and Wellness. New York: Springer; 2012.

6. Neffa JC. Los riesgos psicosociales en el trabajo contribución a su estudio. Ciudad Autónoma de Buenos Aires: CEIL CONICET; 2015.

7. Blanch J. Condiciones de trabajo y riesgos psicosociales bajo la nueva gestión. Formación Continuada a Distancia; 2011.

8. Der-Shin Ke. Overwork, stroke, and karoshi-death from overwork. Acta Neurol Taiwan. 2012;21(2):549.

9. American Heart Association. Mental Health and Heart Health | American Heart Association. https://www.heart.org/en/healthy-living/healthy-lifestyle/mental-health-and-wellbeing/mentalhealth-and-heart-health (2018). Accessed 18 Dec 2019 Dec.

10. Schnall PL, Dobson M, Landsbergis P. Globalization, Work, and Cardiovascular Disease. Int J Heal Serv. 2016;46(4):656-92.

11. Kivimäki M, Kawachi I. Work Stress as a Risk Factor for Cardiovascular Disease. Curr Cardiol Rep. $2015 ; 17(9)$.

12. International Labour Organization. Working Time Arrangements. Geneva: International Labour Office; 2019. 1-48 p.

13. Vahtera J, Kivimäki M, Pentti J, Linna A, Virtanen $M$, Virtanen P, et al. Organisational downsizing, sickness absence, and mortality: 10-Town prospective cohort study. Br Med J. 2004;328(7439):5558.

14. Zhao Y, Richardson A, Poyser C, Butterworth P, Strazdins L, Leach LS. Shift work and mental health: a systematic review and meta-analysis. Vol. 92. Berlin Heidelberg: International Archives of Occupational and Environmental Health. Springer; 2019. 763-93 p.

15. Kervezee L, Kosmadopoulos A, Boivin DB. Metabolic and cardiovascular consequences of shift work: The role of circadian disruption and sleep disturbances. Eur J Neurosci. 2018;00:1-17.

16. Kervezee L, Cuesta M, Cermakian N, Boivin DB. Simulated night shift work induces circadian misalignment of the human peripheral blood mononuclear cell transcriptome. Proc Natl Acad Sci U S A. $2018 ; 115(21): 5540-5$. 
17. Teixeira LR, Azevedo TM, Bortkiewicz A, Corrêa da Silva DT, de Abreu W, de Almeida MS, et al. WHO/ILO work-related burden of disease and injury: Protocol for systematic reviews of exposure to occupational noise and of the effect of exposure to occupational noise on cardiovascular disease. Environ Int. 2019;125(2019):567-78.

18. Babisch W. Cardiovascular effects of noise. Noise Health. 2011;13(52):201-4.

19. Tomei G, Fioravanti M, Cerratti D, Sancini A, Tomao E, Rosati MV, et al. Occupational exposure to noise and the cardiovascular system: A meta-analysis. Sci Total Environ. 2010;408(4):681-9.

20. Woodruff TJ, Sutton P. The navigation guide systematic review methodology: A rigorous and transparent method for translating environmental health science into better health outcomes. Environ Health Perspect. 2014;122(10):1007-14.

21. Moher D, Shamseer L, Clarke M, Ghersi D, Liberati A, Petticrew M, et al. Preferred reporting items for systematic review and meta-analysis protocols (PRISMA-P) 2015 statement. 2015;1-9.

22. ICD-11 - Mortality and Morbidity Statistics. https://icd.who.int/browse11/I-m/en. Accessed $30 \mathrm{Apr}$ 2020.

23. Li J, Brisson C, Clays E, Ferrario MM, Ivanov ID, Landsbergis P, et al. WHO/ILO work-related burden of disease and injury: Protocol for systematic reviews of exposure to long working hours and of the effect of exposure to long working hours on ischaemic heart disease. Environ Int. 2018;119(August):558-69.

24. Babineau J. Product Review: Covidence (Systematic Review Software). J Can Heal Libr Assoc /. J I'Association des bibliothèques la santé du Canada. 2014;35(2):68-71.

25. Moher D, Liberati A, Tetzlaff J, Altman DG. Preferred reporting items for systematic reviews and meta-analyses: The PRISMA statement. BMJ. 2009;339:b2535.

26. Chiu W, Johnson N, Moriarty M, Pulczinsk J, Uwak I, Taiwo S, et al. Applying the Navigation Guide Systematic Review Methodology Case Study \#7: Association between Prenatal Exposures to Ambient Air Pollution and Birthweight. In preparation. 2017.

27. Lam J, Koustas E, Sutton P, C M, Padula EW. A, et al. Applying the Navigation Guide: Case Study \#6. Association Between Formaldehyde Exposures and Asthma. Prep. 2016;(April).

28. Campbell M, Mckenzie JE, Sowden A, Katikireddi SV, Brennan SE, Ellis S, et al. Synthesis without meta-analysis (SWiM) in systematic reviews: reporting guideline. BMJ. 2020;368(16890).

29. McKenzie JE, Brennan SE. Synthesizing and presenting findings using other methods. In: Higgins J, Thomas J, Chandler J, Cumpston M, Li T, Page M, et al., editors. Cochrane Handbook for Systematic Reviews of Interventions. 2nd Editio. Chichester (UK): John Wiley \& Sons; 2019. p. 321-48.

30. Higgins J, Thomas J, Chandler J, Cumpston M, Li T, Page M, et al. Cochrane Handbook for Systematic Reviews of Interventions. 2nd Editio. Chichester: John Wiley \& Sons; 2019.

31. Arena R, Guazzi M, Briggs PD, Cahalin LP, Myers J, Kaminsky LA, et al. Promoting health and wellness in the workplace: $A$ unique opportunity to establish primary and extended secondary cardiovascular risk reduction programs. Mayo Clin Proc. 2013;88(6):605-17. 
32. Moher D, Shamseer L, Clarke M, Ghersi D, Liberati A, Petticrew M, et al. Preferred reporting items for systematic review and meta-analysis protocols (PRISMA-P) 2015 statement. Syst Rev. 2015;4(1).

\section{Figures}

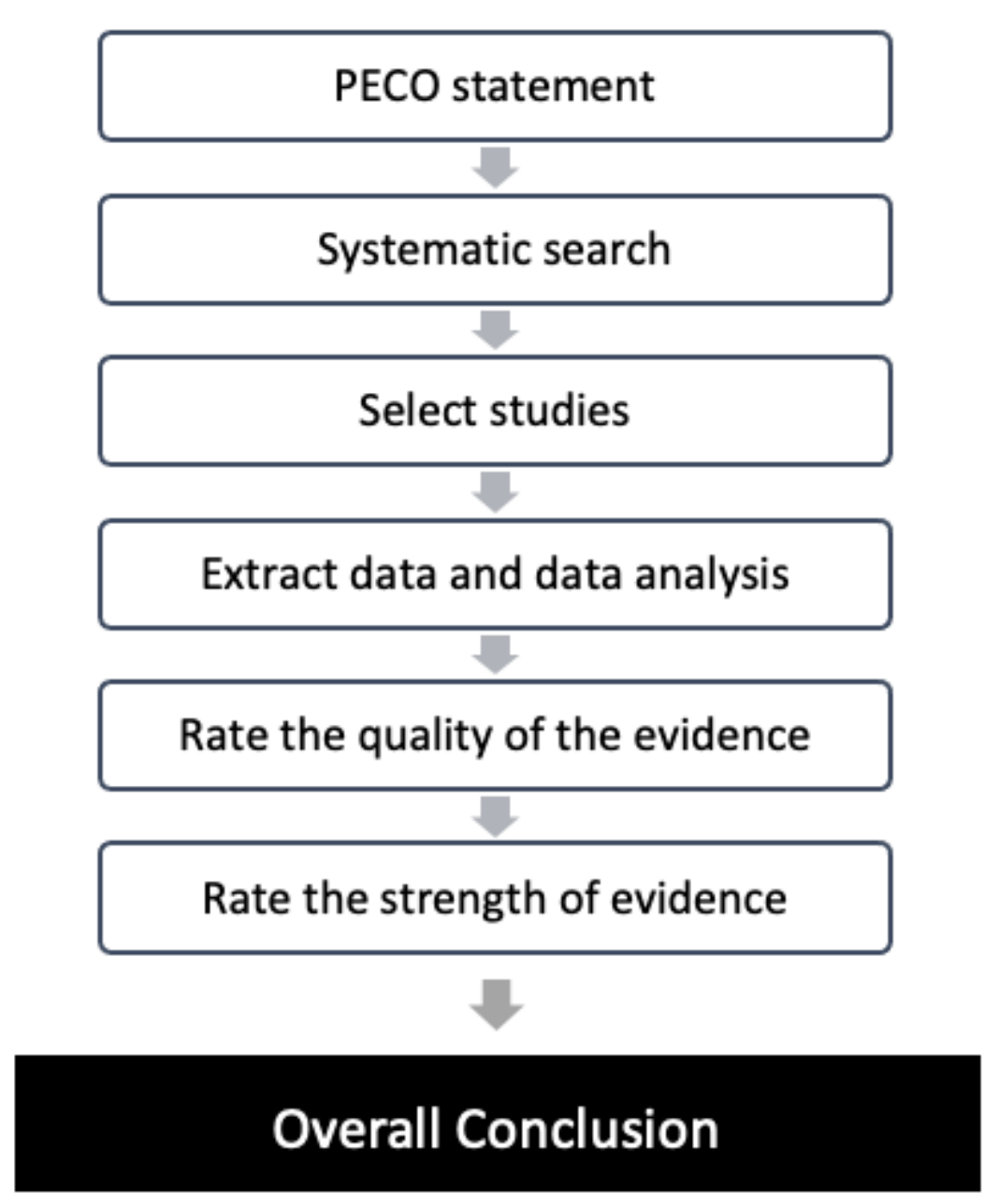

Figure 1

Navigation Guide Steps

\section{Supplementary Files}

This is a list of supplementary files associated with this preprint. Click to download.

- Additionalfile1.docx

- Additionalfile2.docx

- Additionalfile3.docx 
- Additionalfile4.docx

- Additionalfile5.docx

- Additionalfile6.docx 